REVISTA ANDALUZA DE ANTROPOLOGÍA

NÚMERO 15: ACTIVIDADES TURÍSTICAS, CIUDAD Y PATRIMONIO CULTURAL: MIRADAS CRÍTICAS. TOURIST ACTIVITIES, CITY AND CULTURAL HERITAGE: CRITICAL GLANCES SEPTIEMBRE DE 2018

ISSN 2174-6796

[pp. 183-197]

http://dx.doi.org/10.12795/RAA.2018.15.08

\title{
TURISTIFICATION IN LISBON. COLECCIÓN DE DIBUJOS ETNOGRÁFICOS
}

Manuel João Ramos.

CEI-IUL Centro de Estudos Internacionais (CEI-IUL)

Instituto Universitário de Lisboa (ISCTE-IUL) 


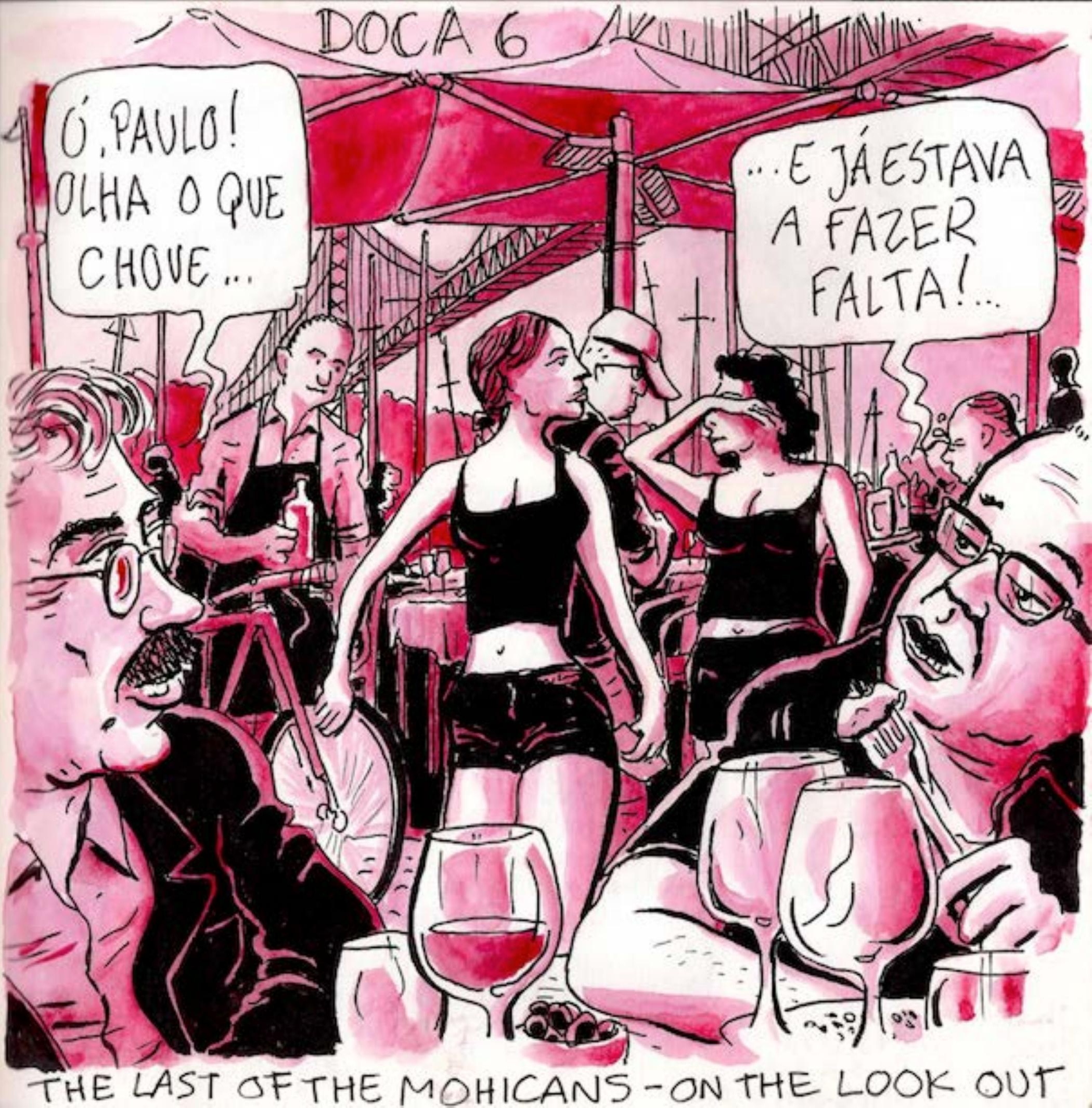




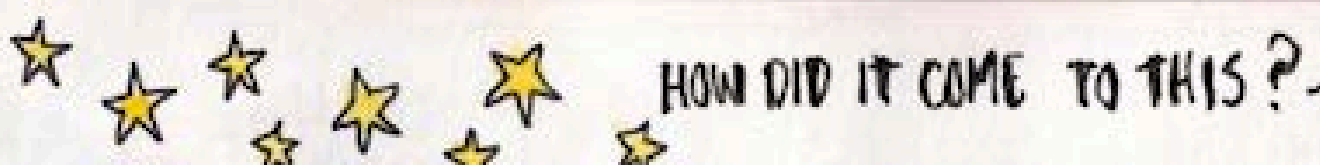

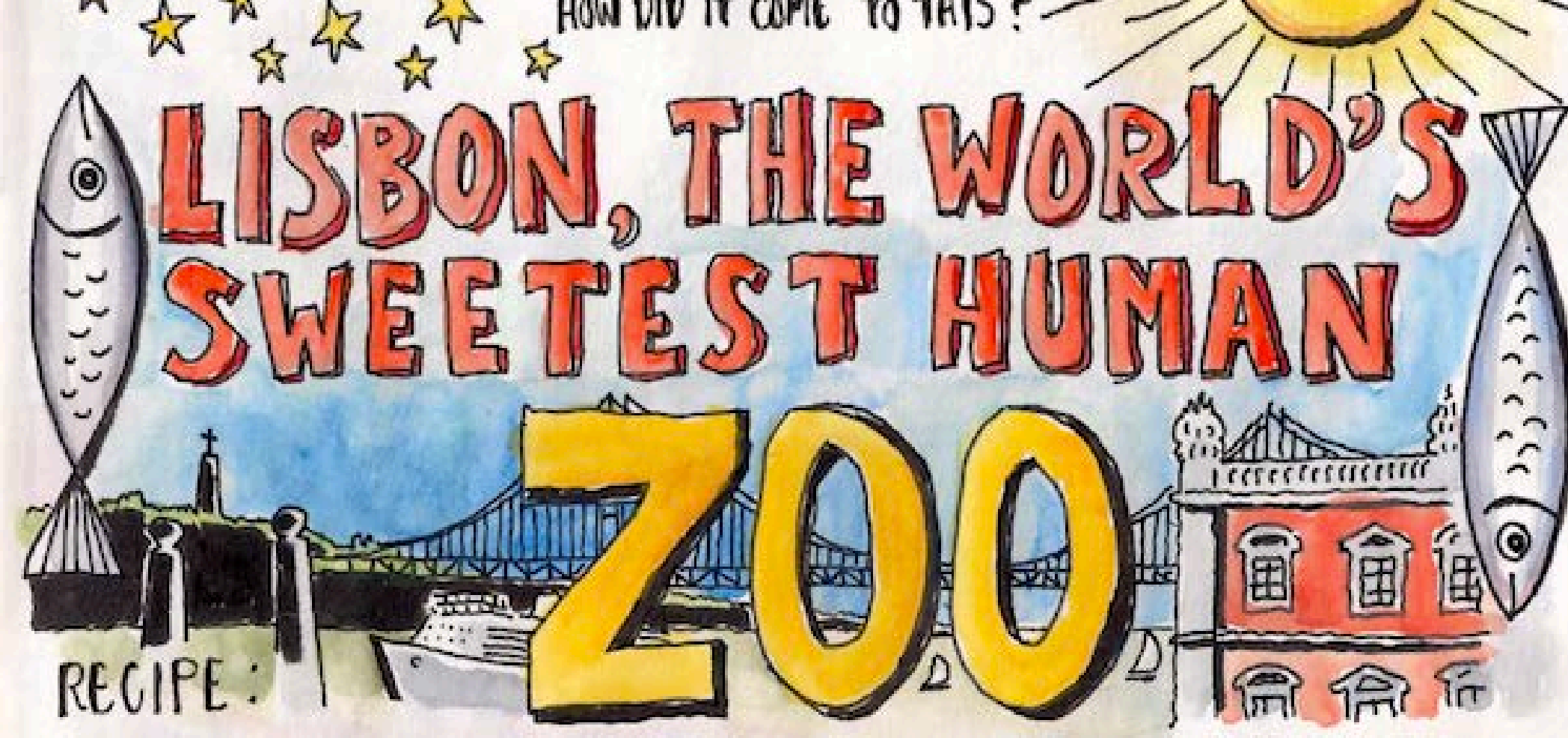

NICE WEATHER, GENTLE PEOPLE, CHEAP FOOD \& DRINKS

MIX IN WELL, SIMMER FOR A WHILE, SERVE WITH A GARNISHMENT OF NEOLIBERAL POLICIES WITH A SOCIAL TOUCH, A BRRCELONA-STYLE DIP, A SHARP DECLINE OF TOURISM IN POLITICALLY -TROUBLED COUNTRIES (WITH THANKS TO THE "ARAB SPRING") AND A SLUSHY OFFER OF GOLDEN VISAS AND EXPAT RETIREMENT SCHEMES.

* SEASON to taSte WITH ERASMUS STUDENTS, LOW-COST FLIGHTS AND CRUISE SHIPS 


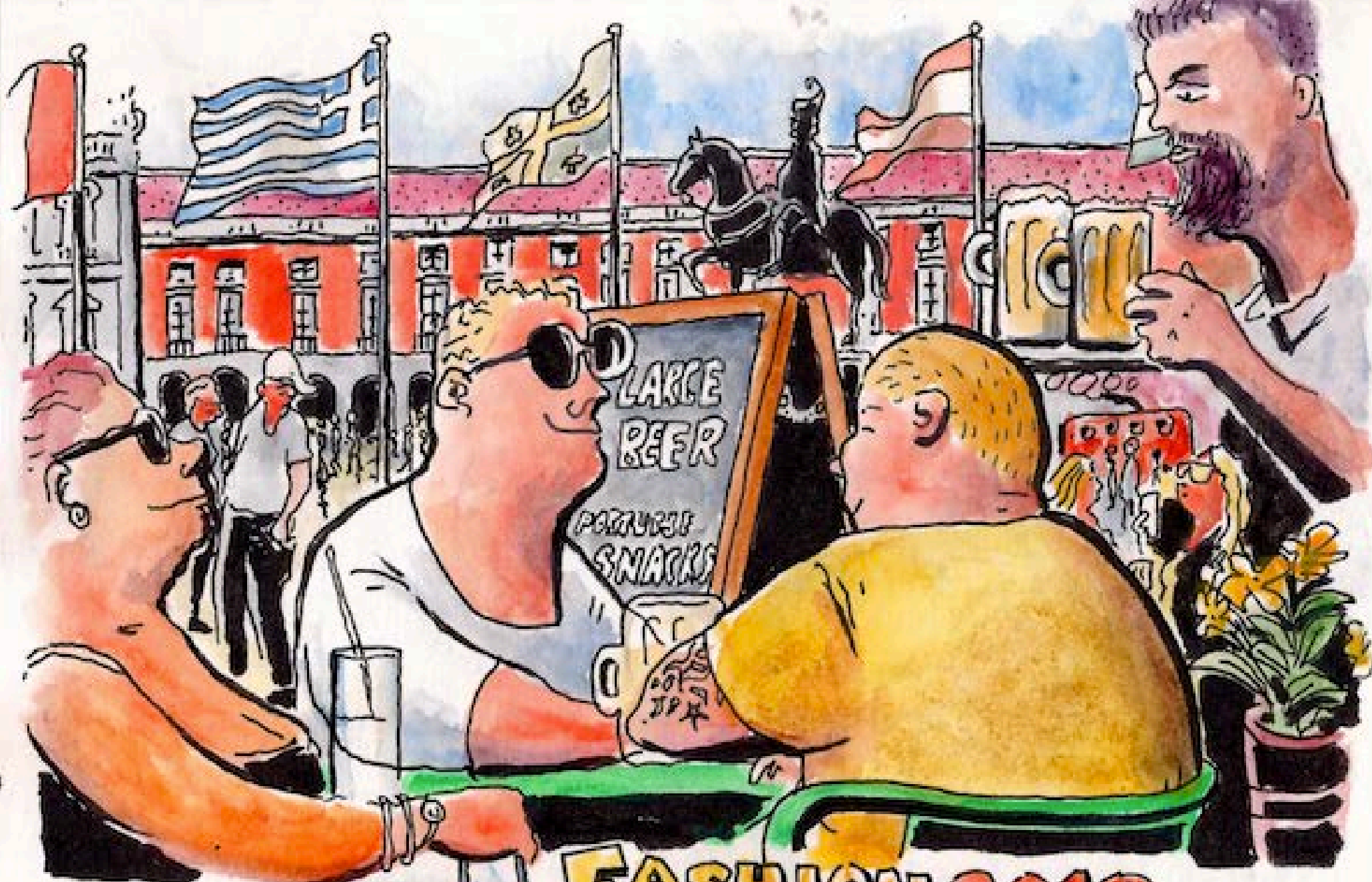

Q FATCION2018 MATERIAL COLTURE (TOURIST GEAR, ADAPTED SPECIALLY FOR TEMPERATE CLIMATS, MIDDLE-INCOME-
COUNTRES, HARROWING SIDE-WALKS): SUNGLASSES

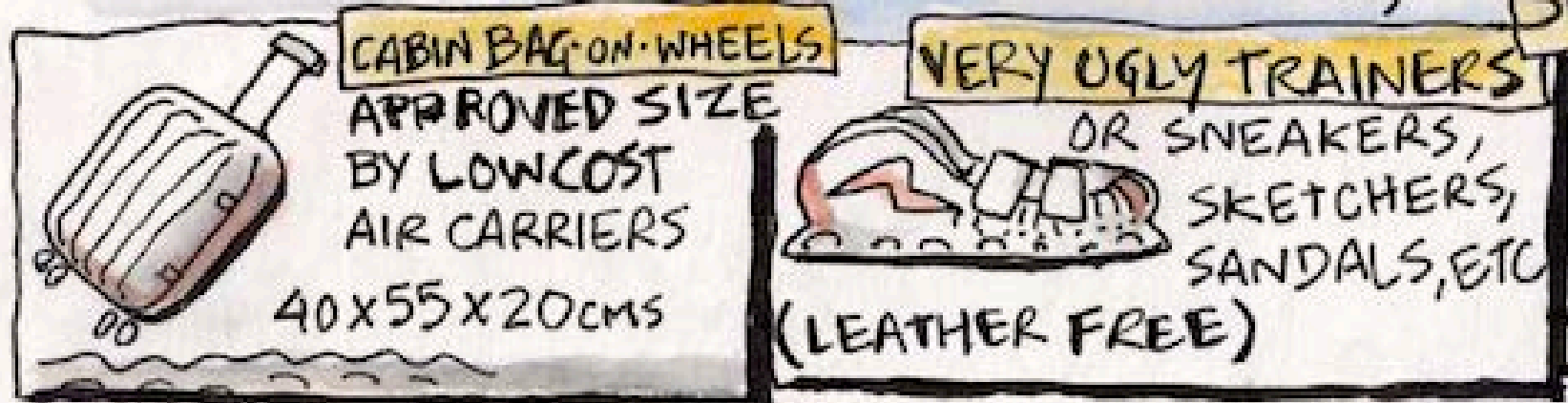

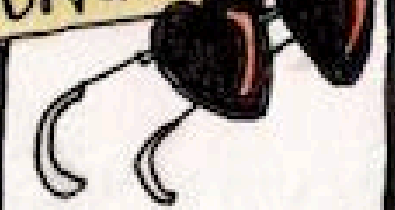
ALL SHAPES ALLOWED 


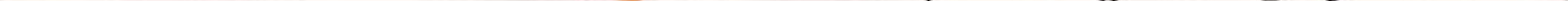




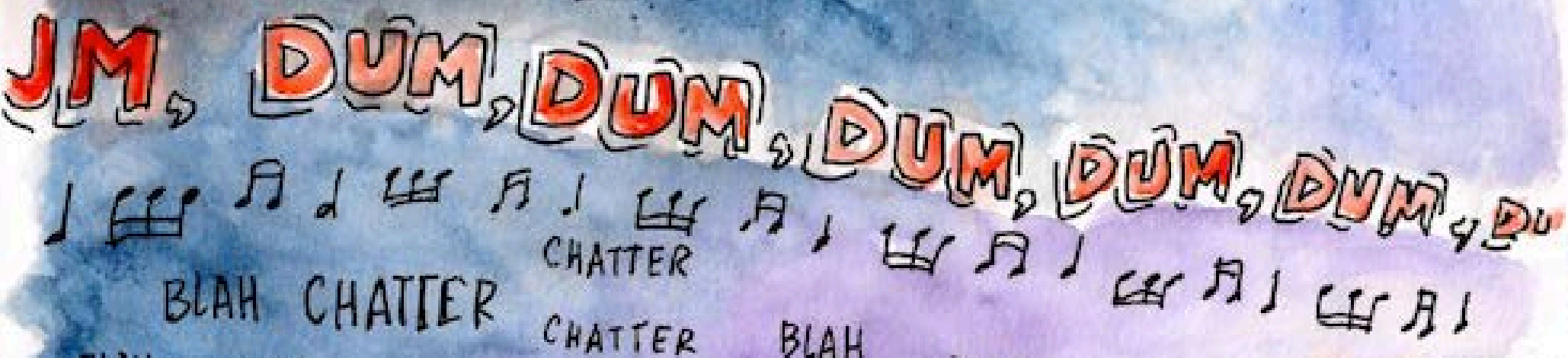

BLAH CHATTER CHATTER BLAH BLAH

BLAH CHATtER CHATTER BLAH CHATTER BLAH
BLAH CHATTER BLAH CHATTER BLAH CHATTER BLAMER CHATTER BLAH

唡 


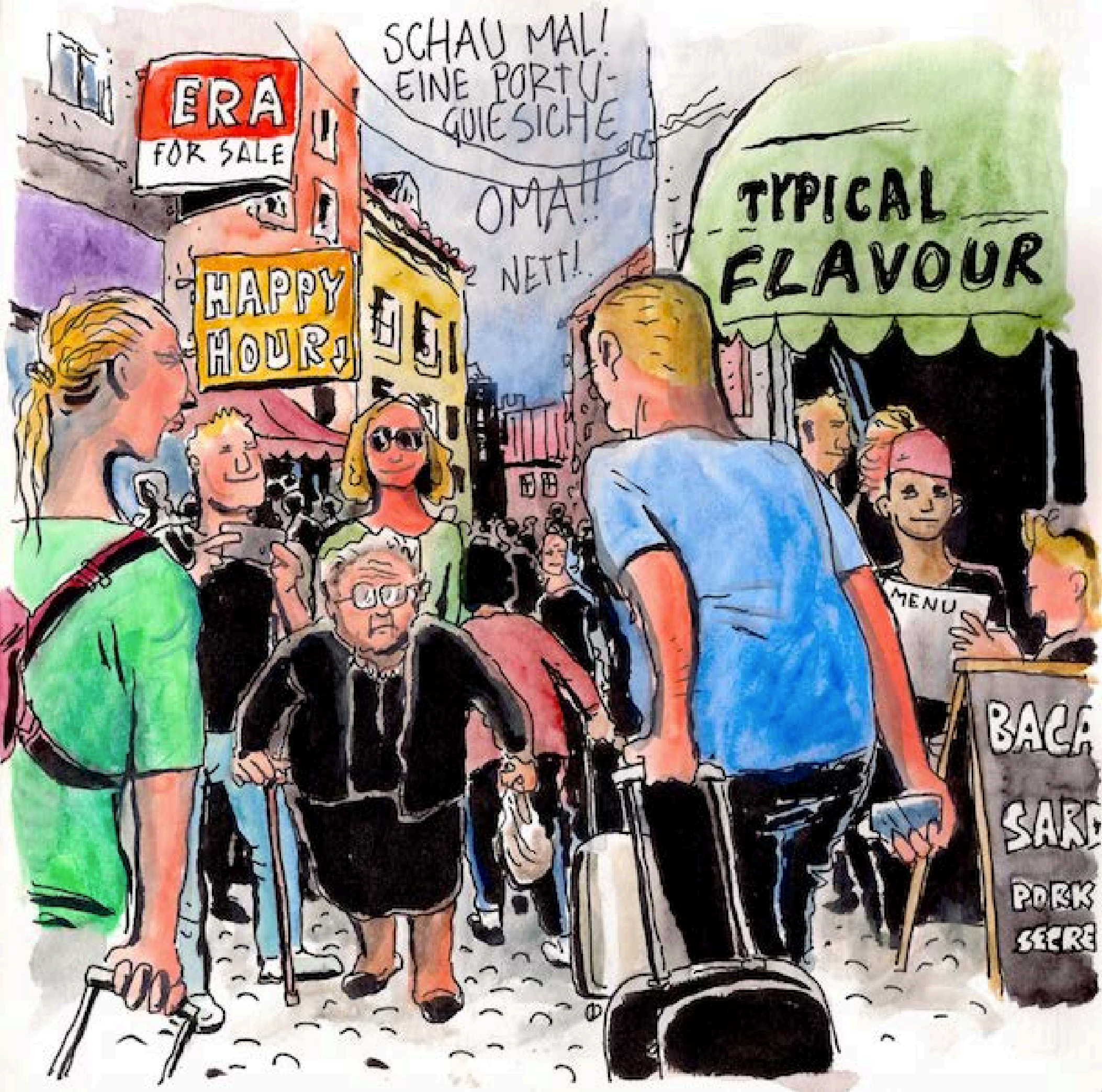



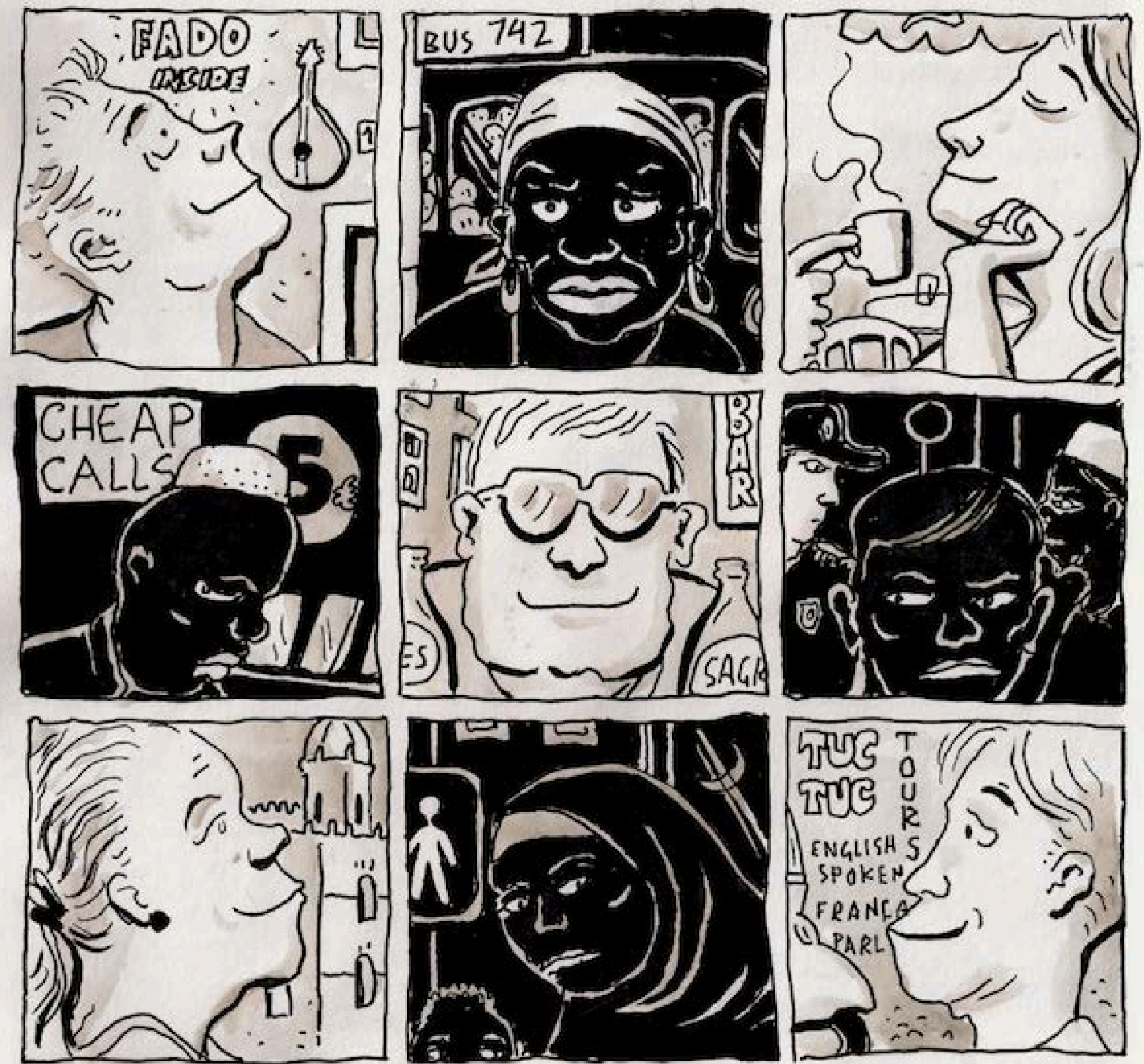


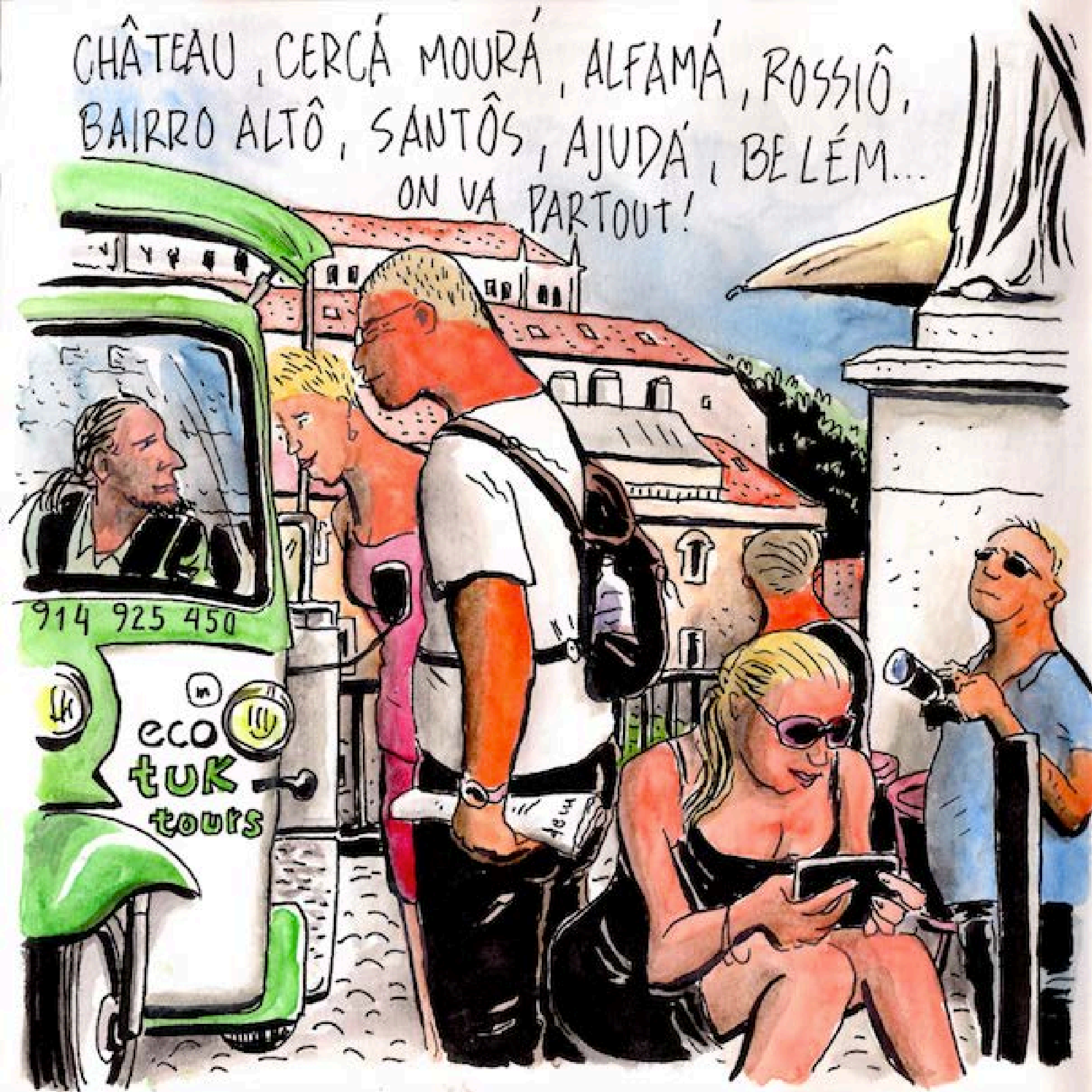




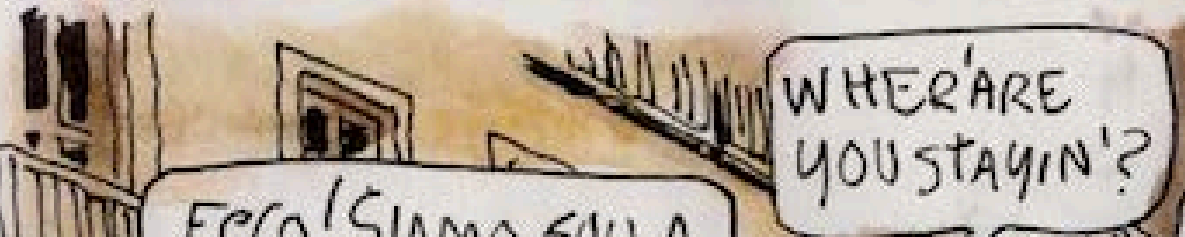

EECO! SIAMO SOLLA
STRADA ROSA.

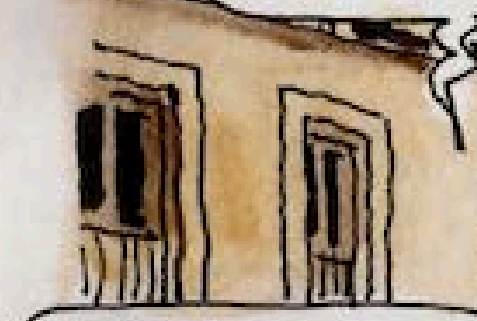

ABER DIE KEUNER

\section{SPRECHEN}

- NUR ENGLISAH!

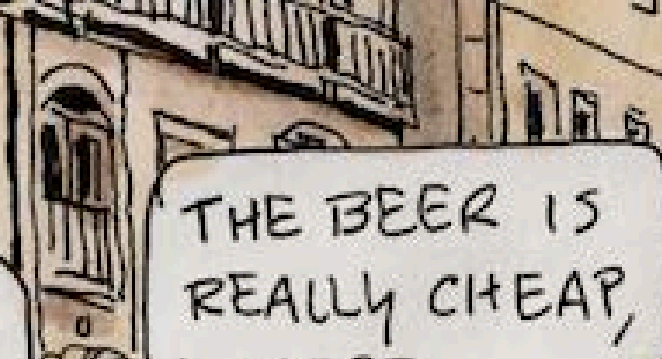

VF HERE...

W $P$ WD WW n

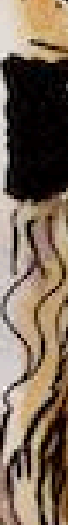
ट

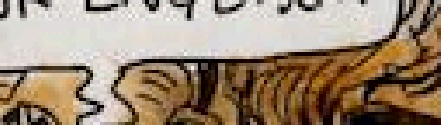

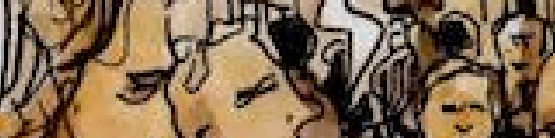

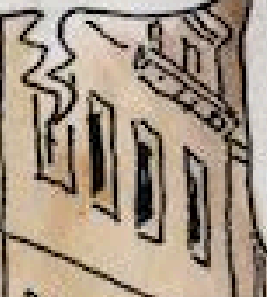

EN ESPAGNE, ON DIT BOTELLÓN. ICI, JE NE SAIS PAS.

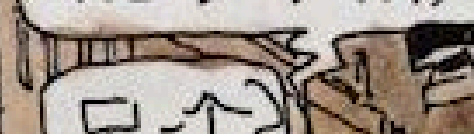
穷-个? 质 गाएग Eरचा HAVE YOU EVER TRIED A KALASHNIKOV? IT HAS ABSYNTH.

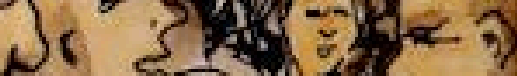
(र)

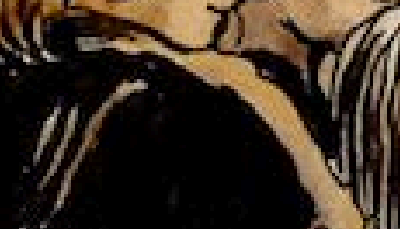
in) 1 
